Enfoques. Revista de Investigación en Ciencias de la Administración http://doi.org/10.33996/revistaenfoques.v4i15.90 No. 15 | Volumen 4 | Julio - Septiembre 2020 enfOQques http://revistaenfoques.org

ISSN: $2616-8219$

ISSN-L: $2616-8219$

pp. $185-191$

\section{INDICADORES DE LA GESTIÓN DE CALIDAD EN LAS EMPRESAS DE FLUIDOS DE PERFORACIÓN}

\author{
INDICATORS OF QUALITY MANAGEMENT IN DRILLING FLUID \\ COMPANIES
}

\author{
INDICADORES DE GESTÃO DA QUALIDADE EM EMPRESAS DE \\ FLUIDOS DE PERFURAÇÃO
}

\author{
José Luis González
}

Artículo recibido marzo 2020 | Arbitrado abril 2020 | Publicado 01 de julio 2020

\section{Resumen}

El presente manuscrito tiene como propósito mostrar y determinar los indicadores de la gestión de calidad que se cumplen en las empresas de fluidos de perforación. La metodología empleada fue descriptiva, con diseño de campo, no experimental y transversal. La información se recolectó a través de un cuestionario contentivo de 12 ítems, con escala de frecuencias. Se llevó a cabo en las empresas de fluido de perforación ubicadas en la Costa Oriental del Lago, Venezuela cuyos informantes fueron los gerentes de operaciones y coordinadores de calidad. El cuestionario fue validado por expertos y la confiabilidad calculada por la formula Alfa de Cronbach, con coeficiente de 0,98. Para procesar los resultados de la aplicación del cuestionario se empleó la media aritmética. Se pudo conocer que estas empresas no hacen medición de su desempeño, pues todos los indicadores capacidad total, capacidad utilizada, servicio al cliente y nivel de cumplimiento fueron valorados en mediano cumplimiento.

Palabras clave: Capacidad total, capacidad utilizada, gestión de calidad, indicadores, nivel de cumplimiento, servicio al cliente

\begin{abstract}
The purpose of this manuscript is to show and determine the indicators of quality management that are met in drilling fluid companies. The methodology used was descriptive, with a field design, non-experimental and cross-sectional. The information was collected through a questionnaire containing 12 items, with a frequency scale. It was carried out in the drilling fluid companies located on the Eastern Coast of the Lake, Venezuela whose informants were the operations managers and quality coordinators. The questionnaire was validated by experts and the reliability calculated by Cronbach's Alpha formula, with a coefficient of 0.98 . To process the results of the questionnaire application, the arithmetic mean was used. It was learned that these companies do not measure their performance, since all indicators of total capacity, used capacity, customer service and level of compliance were assessed as medium compliance.
\end{abstract}

Key words: Total capacity, used capacity, quality management, indicators, level of compliance, customer service

\begin{abstract}
José Luis González
jolugonpe@hotmail.com

ORCID: 0000-0001-9478-6291

Universidad del Zulia. Núcleo Costa Oriental del Lago, Venezuela

Ingeniero Mecánico, egresado de la Universidad del Zulia, Venezuela. Maestría en Gerencia de Empresas, mención Operaciones. Universidad del Zulia.
\end{abstract}




\section{Resumo}

O objetivo deste manuscrito é mostrar e determinar os indicadores de gestão da qualidade que são atendidos em empresas de fluidos de perfuração. A metodologia utilizada foi descritiva, com desenho de campo, não experimental e transversal. As informações foram coletadas por meio de um questionário contendo 12 itens, com escala de frequência. Foi realizado nas empresas de fluidos de perfuração localizadas na Costa Leste do Lago, Venezuela, cujos informantes foram os gerentes de operações e coordenadores de qualidade. 0 questionário foi validado por especialistas e a confiabilidade calculada pela fórmula Alfa de Cronbach, com coeficiente de 0,98. Para processar os resultados da aplicação do questionário, foi utilizada a média aritmética. Apurou-se que estas empresas não medem o seu desempenho, uma vez que todos os indicadores de capacidade total, capacidade utilizada, serviço ao cliente e nível de conformidade foram avaliados como conformidade média.

Palavras-chave: capacidade total, capacidade utilizada, gestão da qualidade, indicadores, nível de conformidade, atendimento ao cliente

\section{INTRODUCCIÓN}

Las organizaciones hoy en día, están enfrentando un proceso de cambio originado por el alto nivel de competencia en los mercados internacionales, debido a la aparición de nuevas tecnologías, productos, competidores, y capacidades. Esta situación se presenta como un reto a la sobrevivencia de la empresa, impulsando el nacimiento de nuevas formas de gestión, orientadas en su mayoría al área de la calidad, la satisfacción del cliente, la participación de los miembros de la organización y la productividad.

En este sentido, es oportuno destacar la importancia de comprender que actualmente las empresas están en un periodo de transición relevante, pues se han convertido en el principal motor de transformación histórica. Por tal razón, en los últimos años ha existido un marcado interés por especialistas de diferentes áreas del saber, relacionadas, directas e indirectamente, con el mundo de los negocios, en desarrollar estrategias, técnicas, teorías o nuevos modelos bien sean estos organizacionales, económicos, contables, y de procesos, que sirvan de herramientas para mantenerse en este estadio llamado globalización.

Desde un punto general, las empresas exitosas y competitivas a nivel mundial, son las que alcanzan y se mantienen en el estadio de la excelencia. Porter (2006) afirma que el ser competitivo hoy en día significa tener características especiales que hacen ser escogidos dentro de un grupo de empresas que se encuentran en un mismo mercado buscando ser los seleccionados.

Al respecto, Moya (2006) señala que el fundamento último de la gestión de calidad es lograr que la empresa sea competitiva y rentable; es decir, que venda de forma rentable. Por tanto, su objetivo es satisfacer las necesidades de los clientes de la forma más rentable y eficiente posible, garantizando que todas las actividades de la empresa contribuyan al logro de sus objetivos.

En este marco de ideas Pérez (2004) plantea que la idea de gestión de calidad lleva implícito el concepto de mejora como un proceso que contribuye a reducir los costos totales y aportando una sólida ventaja competitiva. En este sentido se debe tener presente que, la ventaja competitiva viene siempre de alcanzar altos niveles de satisfacción del cliente, lo cual puede exigir en algunos casos acciones específicas que al no ser repetitivas no estarán recogidas en los procedimientos que formen parte del manual de aseguramiento de la calidad.

Por otra parte Juran (2001), establece que para lograr ventajas competitivas la gestión de calidad posee una trilogía compuesta por tres procesos centrales: planificación de la calidad, donde se determina quienes son los usuarios 
para definir las necesidades que deben cubrir las características de los productos; control de calidad, donde se evalúa el comportamiento real del producto y se compara con sus objetivos a fin de tomar decisiones; y por último el mejoramiento de la calidad, donde se establece la infraestructura, se identifican los proyectos de mejoramiento y se proporcionan los recursos a los equipos.

Ante esta realidad, debido a la gran influencia de empresas extranjeras que llegaron a Venezuela a desarrollar sus productos y servicios, el parque industrial venezolano ha tenido que adaptarse a estas exigencias de calidad, y aunque no todas las organizaciones están adelantadas en este proceso, sí existe la preocupación de diferentes sectores por adecuarse a procedimientos que le aseguren una mayor demanda y preferencia por el público consumidor. A pesar de este boom de calidad, algunas de las empresas del país se han quedado rezagadas en la aplicación y seguimiento de principios de calidad, ejemplo de este caso es el de las empresas de fluidos de perforación, específicamente las ubicadas en la Costa Oriental del Lago del estado Zulia, Venezuela.

Este sector dentro de la economía nacional e internacional, ha venido sufriendo cambios a los cuales se ha visto obligada a adoptar a fin de adaptarse a las exigencias de un mercado cada vez más complejo. Durante mucho tiempo los fluidos de perforación no eran tomados en cuenta como servicio de gran importancia en la perforación de pozos, solo eran utilizados para la extracción de cortes de rocas a la superficie. El siglo XXI se ha caracterizado por presentar un avance en los estudios de utilización de los fluidos de perforación; estos estudios han dado como resultado el descubrimiento de la importancia de los fluidos de perforación para la optimización de producción del pozo.
Ahora bien, los fluidos de perforación se han convertido en uno de los ejes principales de perforación y producción de pozo. Hoy día, además de transportar los ripios a superficie, es uno de los factores principales para evitar fallas en las operaciones de perforación, por lo que los fluidos de perforación deben cumplir con funciones de gran importancia $y$ directamente relacionados con la eficiencia, economía y total computación de la operación de perforación. Todas estas razones han hecho que las exigencias de los clientes vayan aumentando día a día y por ende que los fluidos de perforación y sus propiedades resultantes estén sujetas a muchos estudios y análisis para garantizar la calidad de servicio; es decir, mantener un sistema de gestión de calidad.

Partiendo de estas consideraciones y entendiendo el importante valor agregado que genera para la organización una adecuada gestión de calidad, se hace necesario determinar los indicadores de gestión que se cumplen en las empresas de fluidos de perforación.

\section{MÉTODO}

La investigación estuvo enmarcada dentro de un estudio descriptivo, según el nivel de profundidad con que se estudió la variable objeto de estudio que concuerda con el objetivo de la investigación, y el esquema utilizado para obtener las causas y consecuencias de la gestión de calidad en las empresas de fluido de perforación ubicadas en la Costa Oriental del Lago. Asimismo, el diseño fue de campo, no experimental y transversal.

La información se recolectó a través de un cuestionario contentivo de 12 ítems, con escala de frecuencias. Se llevó a cabo en las empresas de fluido de perforación ubicadas en la Costa Oriental del Lago, cuyos informantes fueron los gerentes de operaciones $y$ 
coordinadores de calidad. El cuestionario fue validado por expertos y la confiabilidad calculada por la formula Alfa de Cronbach, con coeficiente de 0,98 , considerado de muy alta confiabilidad.
Para procesar los resultados de la aplicación del cuestionario se recurrió al método de la estadística descriptiva, y se empleó la media aritmética. En la tabla 1 se establece el baremo para su interpretación.

Tabla 1. Baremo para la interpretación de la media aritmética

\begin{tabular}{lll}
\hline RANGO & CATEGORÍA & DESCRIPCIÓN \\
\hline $2.35-3.00$ & Alto cumplimiento & $\begin{array}{l}\text { Si el cumplimiento es alto, se considera una fortaleza } \\
\text { para la gestión de calidad. }\end{array}$ \\
$1.68-2.34$ & Mediano cumplimiento & $\begin{array}{l}\text { Si el cumplimiento es mediano o bajo, se considera una } \\
\text { debilidad para la gestión de calidad, por tanto requiere } \\
\text { mejoras. }\end{array}$ \\
\hline
\end{tabular}

\section{RESULTADOS}

En la tabla 2, se muestran los resultados obtenidos para la dimensión considerada en este estudio, denominada indicadores de la gestión de calidad derivada del objetivo, en donde se determinan los indicadores de la gestión de calidad que se cumplen en las empresas de fluido de perforación ubicadas en la Costa Oriental del Lago. La misma se dividió en cuatro indicadores: capacidad total, capacidad utilizada, servicio al cliente y nivel de cumplimiento. A continuación, se muestran los hallazgos evidenciados al respecto, tanto para la dimensión como al desglose de cada uno de los indicadores utilizados en su medición.

Tabla 2. Dimensión: Indicadores de la gestión de calidad

\begin{tabular}{lll}
\hline INDICADORES & MEDIA & CATEGORÍA \\
\hline Capacidad total & 1,79 & Mediano cumplimiento/Debilidad \\
Capacidad utilizada & 1,54 & Bajo cumplimiento/Debilidad \\
Servicio al cliente & 2,02 & Mediano cumplimiento/Debilidad \\
Nivel de cumplimiento & 1,85 & Mediano cumplimiento/Debilidad \\
\hline DIMENSIÓN & $\mathbf{1 , 8 0}$ & Mediano cumplimiento/Debilidad \\
\hline
\end{tabular}

Como puede observarse, los indicadores de la gestión de calidad poseen mediano cumplimiento en la gestión que llevan a cabo las empresas analizadas, ratificado con una media de 1,80, lo cual le atribuye a esta gestión debilidades, implicando que la misma requiere mejoras. Al detalle se observa, mediano cumplimiento en cuanto a los indicadores capacidad total $(1,79)$, servicio al cliente $(2,02)$ y nivel de cumplimiento $(1,85)$; mientras, la capacidad utilizada $(1,54)$ se ubica en bajo cumplimiento, conformándose todos estos aspectos en debilidades de su gestión de calidad, requiriendo mejoras en este sentido.

La situación mostrada contradice a Serna (2010) para quien, los indicadores resultan ser 
una manifestación de los objetivos estratégicos de una organización a partir de su misión, y en el plano de la gestión de calidad, en el monitoreo de la auditoría se deben tomar indicadores construidos para todas y cada una de las actividades que llevan a calificar al servicio y a la calidad en el servicio como excelentes.

Al respecto, a criterio del investigador, en las empresas de fluido de perforación ubicadas en la Costa Oriental del Lago, se tiene poco claro el rol que deben jugar estos indicadores para el logro de una efectiva gestión de la calidad, desconociendo tal vez que estos indicadores influyen sobre los indicadores de gestión de toda la organización, pudiendo ser utilizados por la dirección para proponer acciones globales, o para controlar la situación de determinados parámetros del negocio.

Ahora bien, al desglose de los indicadores utilizados para medir la dimensión, de seguido se especifican los resultados obtenidos, mostrados en la tabla 3, la cual recoge el resultado de la dimensión indicadores de la gestión de calidad y de sus indicadores: capacidad total, capacidad utilizada, servicio al cliente y nivel de cumplimiento.

De esta forma, se evidenció mediano cumplimiento para la dimensión en conjunto bajo una media de 1,80 siendo una debilidad de la gestión de calidad, como ya se mencionó, evidenciándose también mediano cumplimiento en los indicadores capacidad total servicio al cliente y nivel de cumplimiento, con mediano cumplimiento en todas las actividades medidas en estos indicadores a través de los ítems aplicados en ellos, resaltando una actividad medida con bajo cumplimiento en la capacidad total, referida a si cuentan con la capacidad necesaria para alcanzar niveles de desarrollo requerido en la prestación del servicio con una media de 1,50, atribuyéndole debilidades a la gestión de calidad en todos los aspectos medidos.

Además se conoció, dan bajo cumplimiento a la capacidad utilizada, donde catalogaron con esta misma categoría todas las actividades medidas en los ítems aplicados (49, 50 y 51), dejando ver debilidades en su gestión de calidad, indicando que la misma requiere mejoras en todos los aspectos relacionados.

Este resultado, de mediano cumplimiento de la dimensión, no alcanzaron a validar a Mora (2011) para quien, los indicadores de gestión, son variables representativas de los procesos que reflejan el grado de su eficacia y eficiencia, en este caso de la calidad, al compararse con valores esperados. Estos indicadores son expresiones cuantitativas del comportamiento o desempeño de toda una empresa o una parte de ella, cuya magnitud al ser comparada con algún nivel de referencia, puede estar señalando una desviación sobre la cual se tomarán acciones correctivas 0 preventivas, según el caso.

Asimismo, no se evidencio congruencia con el investigador cuando afirma, todas las actividades pueden medirse con parámetros (indicadores) que enfocados a la toma de decisiones son señales para monitorear la gestión, así se asegura que las actividades vayan en el sentido correcto y permiten evaluar los resultados de una gestión frente a sus objetivos, metas y responsabilidades. 
Tabla 3. Indicadores de la gestión de calidad

\section{INDICADOR: CAPACIDAD TOTAL \\ $\mathbf{N}^{\circ}$ ÍTEMS}

MEDIA CATEGORÍA

1. La ejecución de los servicios está comprometida con la aplicación de técnicas de calidad.

2,06 Mediano cumplimiento

Cuentan con la capacidad necesaria para alcanzar

2. niveles de desarrollo requerido en la prestación del 1,50 servicio

Examinan la capacidad para establecer objetivos

3. basados en la cultura de la calidad

Debilidad

1,81 Mediano cumplimiento

Promedio Indicador

Debilidad

\section{INDICADOR:CAPACIDAD UTILIZADA}

$\mathbf{N}^{\circ}$ ÍTEMS

Mediano cumplimiento

Utilizan indicadores que permiten medir la satisfacción

4. de los clientes con su capacidad en el servicio prestado

Están en la capacidad de atender servicios de

5. emergencia en operaciones múltiples

Determinan la capacidad utilizada como indicador de

6. gestión de calidad para medir el nivel de capacidad al

que se encuentra operando la empresa

MEDIA CATEGORÍA

1,63 Bajo cumplimiento

1,56 Bajo cumplimiento

Debilidad

1,44 Bajo cumplimiento Debilidad

Promedio Indicador Bajo cumplimiento

INDICADOR: SERVICIO AL CLIENTE

$\mathbf{N}^{\circ}$ ÍTEMS

1,54

Debilidad

Los procesos están orientados a los clientes

7. obteniendo información de la calidad de las operaciones para su grado de satisfacción

MEDIA CATEGORÍA

\section{Miden la eficiencia del servicio en el sistema de gestión}

8. de calidad a través del nivel de satisfacción del cliente

Mediano cumplimiento

Cuando prometen realizar un servicio al cliente en un

9. tiempo determinado lo cumplen

2,06 Mediano cumplimiento

Debilidad

Debilidad

1,88

Promedio Indicador

Mediano cumplimiento

\section{INDICADOR: NIVEL DE CUMPLIMIENTO}

$\mathbf{N}^{\circ}$ ÍTEMS

Utilizan indicadores que permite medir el grado de

10. cumplimiento de los objetivos planificados en el servicio

Manejan indicadores de gestión que permitan medir el

11. nivel de ejecución de los planes de acción Revisan de forma sistemática los planes, comparando

12. resultados con objetivos e implicando a todos los afectados en los cambios necesarios.

\section{Mediano cumplimiento}

Debilidad

2,02

Debilidad

MEDIA CATEGORÍA

MEDIA CATEGORÍA

Mediano cumplimiento

Debilidad

Mediano cumplimiento

Debilidad

1,81 Mediano cumplimiento

Debilidad

Mediano cumplimiento 
CONCLUSIONES

Para el objetivo, en el cual se determinaron los indicadores de gestión de calidad que se cumplen en las empresas de fluido de perforación ubicadas en la Costa Oriental del Lago, se concluye que en estas empresas no hacen medición de su desempeño, pues todos los indicadores de la gestión de calidad que deben aplicarse para medir su eficacia fueron valorados en mediano cumplimiento, con realce de la capacidad utilizada medida con bajo cumplimiento, representando esta situación debilidades de la gestión, por lo cual se requieren mejoras.

\section{REFERENCIAS}

Juran, J. (2001). Juran y la calidad por el diseño. Edición llustrada. Ediciones Díaz de Santos. México
Mora, A. (2011). Indicadores de la gestión logística. Tercera Edición. Ediciones ECO. Bogotá. Colombia

Moya, J. (2006). Estrategia gestión y habilidades directivas. Edición Ilustrada. Ediciones Díaz de Santos. México

Pérez, J. (2004). Gestión de calidad orientada a los procesos. Edición Ilustrada. ESIC Editorial. Madrid, España

Porter, M. (2006). Ser competitivo. Nuevas aportaciones y conclusiones. Editado por Deusto. Madrid, España

Serna, H. (2010). Gerencia estratégica: teoría, metodología, alineamiento, implementación y mapas estratégicos. Décima edición. 3R Editores. Colombia 